\title{
Single and Double Hydroboration of B-B Triple Bonds and Conver- gent Routes to a Cationic Tetraborane
}

\author{
Tobias Brückner, ${ }^{1,2}$ Tom E. Stennett, ${ }^{1,2}$ Merlin He$\aleph^{1,2}$ and Holger Braunschweig ${ }^{1,2 *}$ \\ ${ }^{1}$ Institut für Anorganische Chemie, Julius-Maximilians-Universität Würzburg, Am Hubland, 97074 Würzburg (Germany), E- \\ mail: h.braunschweig@uni-wuerzburg.de \\ ${ }^{2}$ Institute for Sustainable Chemistry \& Catalysis with Boron, Julius-Maximilians-Universität Würzburg, Am Hubland, 97074 \\ Würzburg (Germany)
}

KEYWORDS Boron, Hydroboration, Cation, Multiple Bonds, Synthesis

\begin{abstract}
A compound with a boron-boron triple bond is shown to undergo stepwise hydroboration reactions with catecholborane to yield an unsymmetrical hydro(boryl)diborene and a 2,3-dihydrotetraborane. Abstraction of $H^{-}$from the latter compound produces an unusual cationic, planar tetraborane with a hydrogen atom bridging the central $B_{2}$ moiety. Spectroscopic and crystallographic data and DFT calculations support a 'protonated diborene' structure for this compound, which can also be accessed via direct protonation of the corresponding diborene.
\end{abstract}

INTRODUCTION The study of boron hydrides has a rich history dating back to the early $20^{\text {th }}$ century, when Alfred Stock's pioneering research on the highly reactive cluster compounds led him to effectively invent the inert atmosphere techniques underpinning much of modern inorganic and organometallic synthesis. ${ }^{1}$ Conclusive determination of the structures of these higher boranes upon the advent of X-ray crystallography ${ }^{2}$ resulted in the classification of "non-classical" multicentre bonding, and boron cluster chemistry grew into an established field in its own right. ${ }^{3-4}$ Classical, two-centre-two-electron bonds between boron atoms are comparatively rare, while welldefined chains of more than two boron atoms are limited to a handful of compounds. ${ }^{5-8}$ One of the more successful routes to such compounds thus far has involved addition reactions with unsaturated diboron species. In 2014 we reported on the hydroboration of diborenes with catecholborane (HBcat) to form linear triboranes, ${ }^{9}$ while the equivalent reactions with 9-borabicyclo[3.3.1] nonane (9-BBN) produced cluster-like triboranes bearing three-centre-two-electron $(3 \mathrm{c} 2 \mathrm{e})$ bonds. ${ }^{10}$ Both our group $^{11}$ and that of Kinjo ${ }^{12}$ have recently demonstrated the diboration of diborenes with $\mathrm{B}_{2} \mathrm{cat}_{2}(\mathrm{cat}=$ catecholato $)$ to form tetraboranes, including in the former case the first triborylboranes. We also recently reported the formation of unsaturated $\mathrm{B}_{4}$ chains in the form of diboryldiborenes via reductive coupling of carbene-stabilized 1,1-dibromodiboranes, ${ }^{13}$ and this class of compound also proved to be accessible in improved yields by the diboration of boron-boron triple bonds with $\mathrm{B}_{2} \mathrm{cat}_{2}$ and $\mathrm{B}_{2} \mathrm{Scat}_{2}$ (Scat $=$ thiocatecholato $) .{ }^{14}$

Over the last decade or so, significant attention has been paid to the relationship between hydrodiboranes(6) that do not contain a formal boron-boron bond (i.e. $\mathrm{B}_{2} \mathrm{H}_{6}$ derivatives) and their classically B-B-bonded diborane(4) and diborene counterparts (Fig. 1). Matsuo, Tamao and co-workers first reported the synthesis of stable diborane $(6)$ dianions $\left[(\mathrm{R}) \mathrm{H}_{2} \mathrm{~B}-\mathrm{BH}_{2}(\mathrm{R})\right]^{2-}(\mathbf{B}$, Scheme 1) via two-electron reduction of diboranes $(\mathrm{R}) \mathrm{HB}(\mu-$
$\mathrm{H})_{2} \mathrm{BH}(\mathrm{R})(\mathbf{A})$, where $\mathrm{R}$ is an extremely bulky aryl group. ${ }^{15-16}$ The same groups reported the abstraction of a hydride from one of these dianions to yield a monoanionic, hydride-bridged diborane(5) (C), which can be treated with $\mathrm{Me}_{3} \mathrm{SiOSO}_{2} \mathrm{CF}_{3}$ to yield hydride-bridged diboranes(4). ${ }^{16}$ Wagner and coworkers then showed that such monoanions, $\mathbf{C}$, (and indeed their diborane(6) precursors) can be reduced to dianionic diborenes $\left[\mathrm{B}_{2} \mathrm{R}_{4}\right]^{2-}(\mathrm{D})$ by using alkali metals to isolate a biphenylene-bridged diborene. ${ }^{17-19}$

The inherent electron deficiency of boron in diboron compounds can also be satiated by neutral Lewis bases. For example, neutral diborenes of the form $\mathrm{L}(\mathrm{R}) \mathrm{B}=\mathrm{B}(\mathrm{R}) \mathrm{L}(\mathrm{I})$ are now accessible with a wide variety of bases and covalent substituents via Wurtz coupling of dihaloboranes or reduction of dihalodiboranes. $^{5}$ Lewis base-stabilized dicationic, hydrogen bridged diboranes $(\mathbf{F})$, formally dimers of borenium ions, have been reported by Curran and Vedejs ${ }^{20}$ and Ghadwal. ${ }^{21} \mathrm{~A}$ handful of neutral, base-stabilized dihydrodiboranes(4) (G) is known, ${ }^{22-26}$ though this is likely to increase with the recent development of transfer hydrogenation to diborenes. ${ }^{27}$ Very recently, the group of Yamashita reported the formation of a hydrogen-bridged tetraborane $(6)\left(\mathbf{E}, \mathrm{R}=\mathrm{BR}_{2}\right)$ upon reductive coupling of the corresponding 1,1-dibromodiborane, presumably via radical hydrogen abstraction by a low-valent $\mathrm{B}_{4}$ intermediate. ${ }^{28}$ Surprisingly, this compound was shown to retain a significant degree of boron-boron multiple bonding between the central boron atoms, suggesting the contribution of a "doubly protonated diboryne" resonance structure. Herein, we report the preparation of a dihydrotetraborane via double hydroboration of a boron-boron triple bond and its subsequent conversion to a cationic tetraborane(5). As well as displaying an interesting bonding situation in its own right, this latter compound represents a further synthetic bridge between key classes of diboron compounds. 


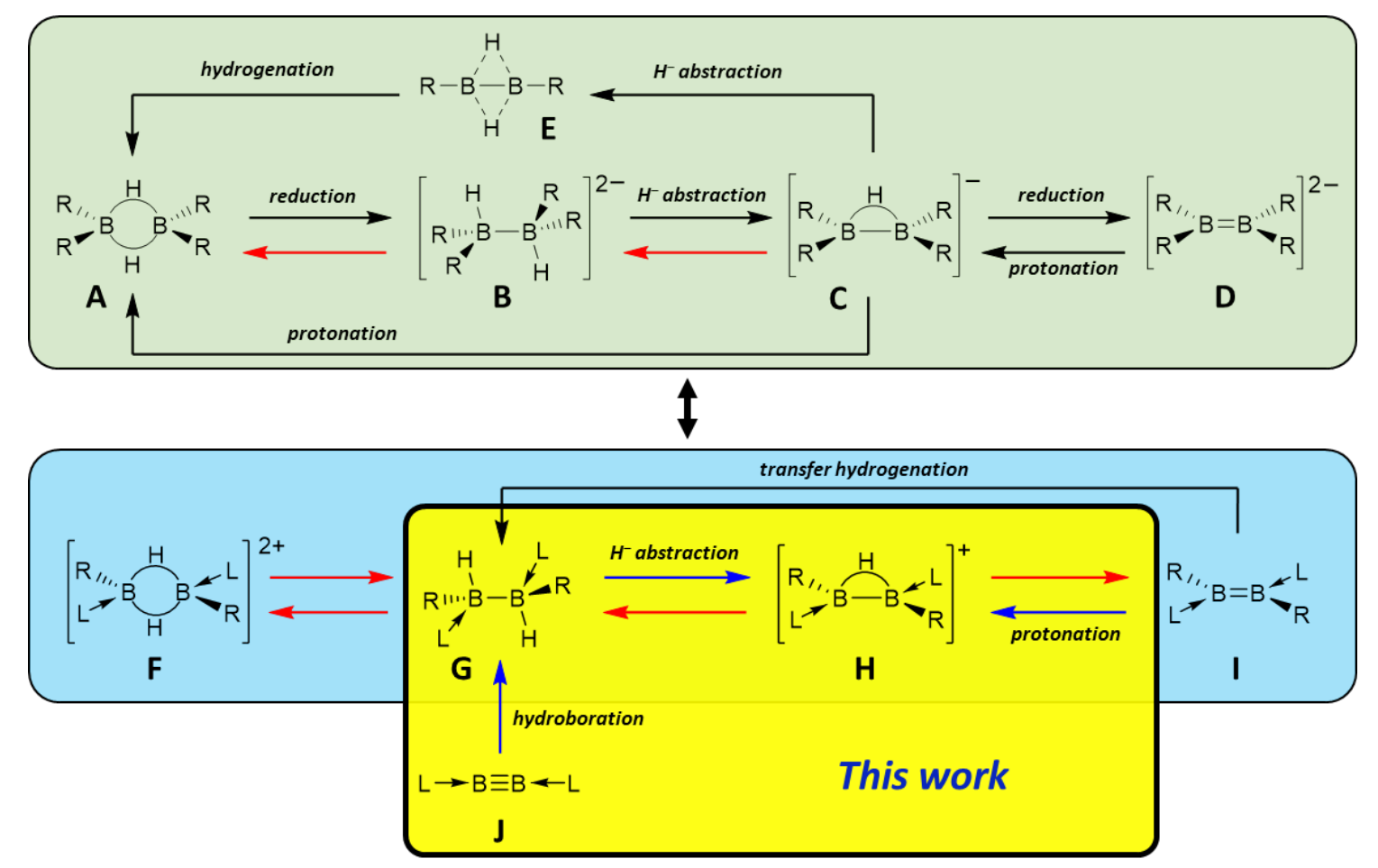

Scheme 1. Isolated diboron hydrogen compounds. Arrow key: black = literature reported transformations; red = as yet unknown transformations; blue $=$ reactions reported herein.

The availability of a series of species with boron-boron triple bonds ( $\mathbf{J}$, Scheme 1) led us to consider the possibility of multiple hydroboration steps at the same molecule as a route to novel tetraboranes. Despite the highly reducing nature of diborynes, the first of which, (IDip)BB(IDip) (1 ${ }^{\text {IDip }}$, IDip = 1,3-bis(2,6diisopropylphenyl)imidazol-2-ylidene), was reported in 2012, ${ }^{29}$ their reactivity is tempered by the shielding effect of the bulky carbene ligands. Small reductions in steric bulk and enhancement of the $\pi$-acceptor character of the NHC have both been found to enhance the reactivity. For example, while $\mathbf{1}^{\text {IDip }}$ could not be hydrogenated to its corresponding dihydrodiborene, replacing the bulky IDip carbenes with the more $\pi$-acidic and somewhat less bulky ligand SIDep (= 1,3-bis(2,6-diethylphenyl)imidazolin-2-ylidene) allowed clean addition of $\mathrm{H}_{2}$ across the B-B triple bond at $80{ }^{\circ} \mathrm{C} .{ }^{30} \mathrm{We}$ chose the diboryne $\mathbf{1}^{\text {SIDipMes }}$ (Scheme 2) for the reactivity study towards catecholborane on the basis of its high reactivity towards $\mathrm{B}_{2} \mathrm{cat}_{2}$ in diboration reactions. ${ }^{14}$

RESULTS AND DISCUSSION Treatment of $1^{\text {SIDipMes }}$ with HBcat in benzene solution led to an immediate color change from red to purple. New signals in the ${ }^{11} \mathrm{~B}$ NMR spectrum at 36.7 and 18.9 ppm suggested formation of diborene 2 , which was isolated as a red powder after workup in $32 \%$ yield. The boron-bound hydrogen atom was observed in the ${ }^{1} \mathrm{H}$ NMR spectrum upon ${ }^{11} \mathrm{~B}$ decoupling as a broad signal at $4.27 \mathrm{ppm}$. The ${ }^{11} \mathrm{~B}$ NMR signal for the Bcat group could not be unambiguously identified due to broadening and overlap with that of the central boron atom. The structure of compound $\mathbf{2}$ was determined by $\mathrm{X}$-ray diffraction on single crystals grown from a saturated pentane solution (Figure 1). At 1.609(2) Å, the diborene B1-B2 distance is similar to that of previously reported diboryldiborenes (1.582-1.627 $\AA$ ). The Bcat group is twisted significantly out of the diborene plane, as shown by the B-B-B-O torsion angle of $62.4(2)^{\circ}$, which suggests that there is negligible conjugation across the $\mathrm{B}_{3}$ unit in the solid state. This is in contrast to the situation for certain diboryldiborenes with smaller NHCs and probably originates from the steric bulk of the mesityl and diisopropylphenyl substituents. Conversely, the NHC at the H-substituted boron atom sits approximately coplanar with the diborene unit. These observations were supported by computation of the frontier orbitals by DFT (B3LYP/6-311G(d); Figure 2); the $\mathrm{HOMO}$ represents the $\mathrm{HB}=\mathrm{B}(\mathrm{Bcat}) \pi$-bond, whereas the LUMO is of $\mathrm{B}=\mathrm{B} \pi$-antibonding character and describes the backbonding from the $\mathrm{B}_{2}$ unit to the NHCs, as expected for diborenes. Unsymmetrical diborenes are still relatively uncommon, ${ }^{31-32}$ and further reactivity studies on $\mathbf{2}$ will be the subject of future work.

Scheme 2. Single and double hydroboration of compound $\mathbf{1}^{\text {SIDipMes }}$, and hydride abstraction from 3 to form the salt 4. 


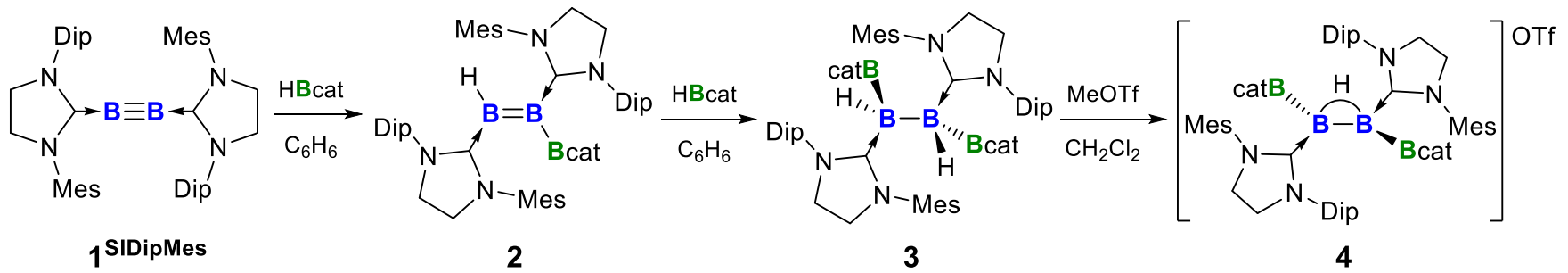

Dip = 2,6-diisopropylphenyl

Diborenes have been shown to react with HBcat to yield hydroboration products, ${ }^{9-10}$ so we therefore treated $1^{\text {SIDipMes }}$ with two equivalents of HBcat in the hope of achieving a second hydroboration. After the initial color change to violet indicating formation of $\mathbf{2}$, colorless crystals began to form within one minute. X-ray diffraction confirmed the formation of tetraborane 3 (Figure 3). Only the linear $\mathrm{B}_{4}$-product was observed, indicating that the second HBcat addition occurs regioselectively. The observation of a single set of NMR signals for $3\left(\delta_{11 \mathrm{~B}}=45.2\right.$ (Bcat), $-33.8(\mathrm{BH})$ ) also confirms the diastereoselectivity of the reaction in favor of the meso product. Compound 3 is a rare example of a tetraborane bound by classical two-centre two-electron covalent bonds. The central boron-boron distance is very large, at 1.842(2) $\AA$, to the best of our knowledge exceeded among neutral, unsupported B-B bonds only by two related, phosphine-stabilized tetraboranes. ${ }^{11}$ The IR spectrum of $\mathbf{3}$ shows a signal for the B-H stretch at $2366 \mathrm{~cm}^{-1}$, which is shifted to higher frequency compared to related anionic derivatives. ${ }^{16}$ DFT calculations showed that the HOMO of 3 represents the central B2-B2' $\sigma$-bond with some distortion towards the hydrogen atoms, confirming the expected electron richness of this moiety.

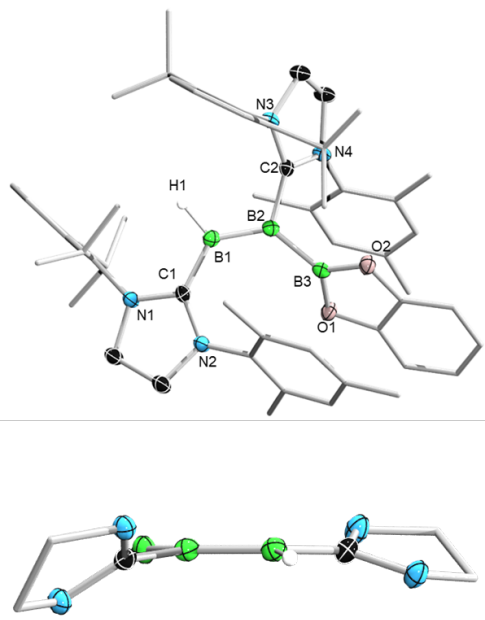

Figure 1. Molecular structure of 2 with selected atomic displacement ellipsoids at $50 \%$ probability displaying the entire molecule (top) and a side view (bottom) showing the planarity of the $\mathrm{B}_{3}-\mathrm{NHC}$ unit. All hydrogen atoms except $\mathrm{H} 1$ are omitted for clarity. Selected bond lengths $(\AA)$ and angles $\left({ }^{\circ}\right)$ : B1-B2 1.609(2), B2-B3 1.690(2), B1-C1 1.553(2), B2-C2 1.571(2), B1-H1 1.15(1), B1-B2-B3 133.4(1), B1-B2-C2 116.9, B2-B1-C1 128.9(1).

Compound 3 reacted with a slight excess of methyl trifluoromethanesulfonate (MeOTf) at room temperature with immedi- ate gas formation. The isolated colorless solid was recrystallized from a dichloromethane/pentane mixture, allowing confirmation of the structure as that of cationic tetraborane $\mathbf{4}$ by single-crystal X-ray diffraction (Figure 3 ). The most notable aspect of the structure is that the four boron atoms lie in a single plane, with the catechol groups at each terminal boron also aligned to allow overlap of the empty p-orbitals of the Bcat groups with the central B-B unit. Conversely, the B1-B2 bond lengths of $1.699(5) \AA$ are in the range of typical catB-B $\left(s p^{2}\right)$ bonds and do not suggest any significant degree of $\pi$-bonding between the atoms. The single remaining hydride was located in the Fourier difference map as bridging between B1 and B1' (supported by other analytical techniques, see below). The B1$\mathrm{B} 1$ ' bond is dramatically shorter than in precursor 3, at 1.635(5) $\AA$, placing it in the range of diborene radical cations. The distance is also somewhat shorter than that in related neutral diboranes(5) (1.670(3) - 1.682(3) $\AA) .{ }^{33-34}$ IR spectroscopy confirmed the absence of terminal B-H bonds, supporting the X-ray structure, although the stretch associated with the bridging B-H-B unit, expected to fall in the fingerprint region of the spectrum, ${ }^{16}$ could not be unambiguously identified.

Compound 4 exists as two isomers in solution in $\mathrm{CD}_{2} \mathrm{Cl}_{2}$ as judged by ${ }^{1} \mathrm{H}$ NMR spectroscopy. The signals for the minor isomer appear broadened at $298 \mathrm{~K}$, but sharpen significantly on cooling to $268 \mathrm{~K}$, while the signals corresponding to the major species are largely unaffected by this temperature change. Measurement of a ${ }^{1} \mathrm{H},{ }^{1} \mathrm{H}$-ROESY spectrum at $268 \mathrm{~K}$ allowed observation of through-space coupling between the respective mesityl and diisopropylphenyl residues, indicating anti (4a, major) and syn (4b, minor) conformers of the compound with respect to the orientation of the N-heterocyclic carbenes (see Supporting Information for further details). Neither heating to 60 ${ }^{\circ} \mathrm{C}$ nor UV irradiation resulted in an alteration of the ratio of isomers. 

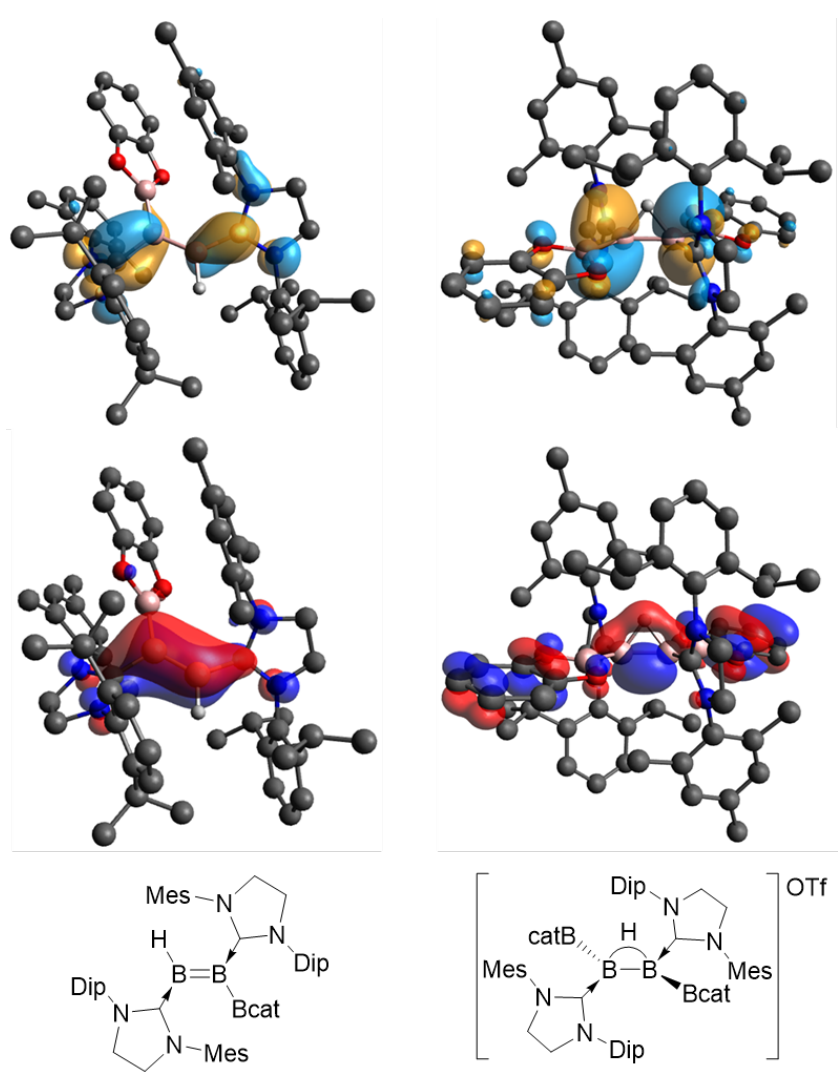

2

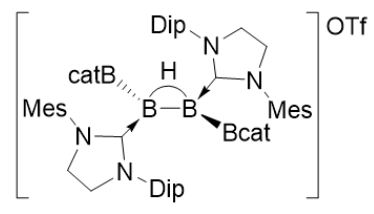

4

Figure 2. Calculated LUMOs (top) and HOMOs (bottom) of compounds 2 (left) and 4 (right) (B3LYP/6-311G(d)).

We carried out DFT calculations at the B3LYP/6-311G(d) level in order to better understand the bonding situation in 4. Optimization of the structure confirmed that the planar configuration represents an energetic minimum with the hydrogen atom

bridging the two central boron atoms. The frontier orbitals are displayed in Figure 2. The HOMO (and, to a lesser extent, the HOMO-8) is represented partially by lobes of pseudo- $\pi$-symmetry across the B-H-B unit, indicating involvement of the hydrogen atom in a 3c-2e bond. This motif was predicted by Lammertsma and Ohwada for hydrogen-bridged compounds ${ }^{35}$ and was observed by Yamashita and co-workers in their tetraborane(6) compound with bridging hydrogen atoms. ${ }^{28}$ Furthermore, neutral diboranes(5) with a bridging hydrogen atom display an almost identical bonding mode across the B-H-B unit. ${ }^{34}$ The LUMO describes the $\pi$-bonding orbitals of the external B1B2 units and is $(\pi)$ antibonding between B1 and B1', resembling that of diboryldiborenes and Yamashita and co-workers' linear tetraborane(6). The energetic difference between the frontier orbitals is large, at $4.586 \mathrm{eV}$, which explains the colorless nature of 4 , in contrast to both diborenes and diborene radical cations. 5 , ${ }^{36}$ The calculated Wiberg bond indices (WBIs) reveal a central boron-boron bond order of 1.131, indicating a modest degree of multiple bonding character, while the calculated WBI values for the $\mathrm{B} 1-\mathrm{H} 1$ and $\mathrm{B} 1$ '-H1 bonds are 0.458 and 0.473 , respectively. The external B1-B2 bonds in 4 have calculated WBI values of 0.956 and 0.962 , respectively, and are thus in the expected range for single bonds. Natural population analysis (NPA) gives partial charge values of -0.221 and -0.264 for $\mathrm{B} 1$ and $\mathrm{B} 1$ ', respectively, and 0.158 for $\mathrm{H} 1$, suggesting a somewhat polar $\mathrm{B}-\mathrm{H}$ bonding interaction. Of the possible resonance forms to describe the bonding in 4 (Scheme 3), this suggests a significant contribution from a "protonated diborene" structure, which is consistent with the crystallographically determined short B-B distance and the ${ }^{11} \mathrm{~B}$ NMR chemical shift of 24.9 ppm. Conversely, the ${ }^{1} \mathrm{H}$ NMR chemical shift of the bridging hydrogen atom at -2.39 (4a) and $-2.32 \mathrm{ppm}(\mathbf{4 b})$ is shifted dramatically upfield compared to Matsuo and Tamao's anionic diborane(5) $(\delta=2.94 \mathrm{ppm})^{16}$ and other neutral derivatives, ${ }^{34}$ being more akin to that of anionic derivatives ${ }^{17,19}$ and suggestive of high shielding.

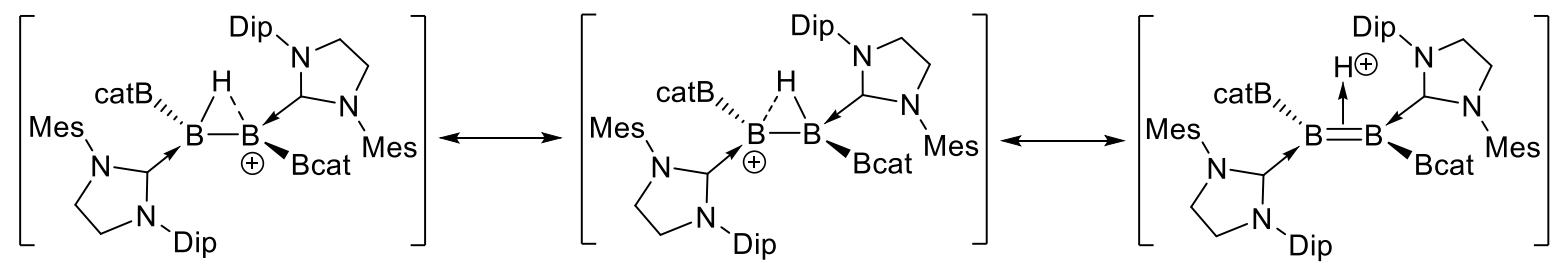

Scheme 3. Possible resonance forms for compound 4. DFT calculations favor the right hand structure. 


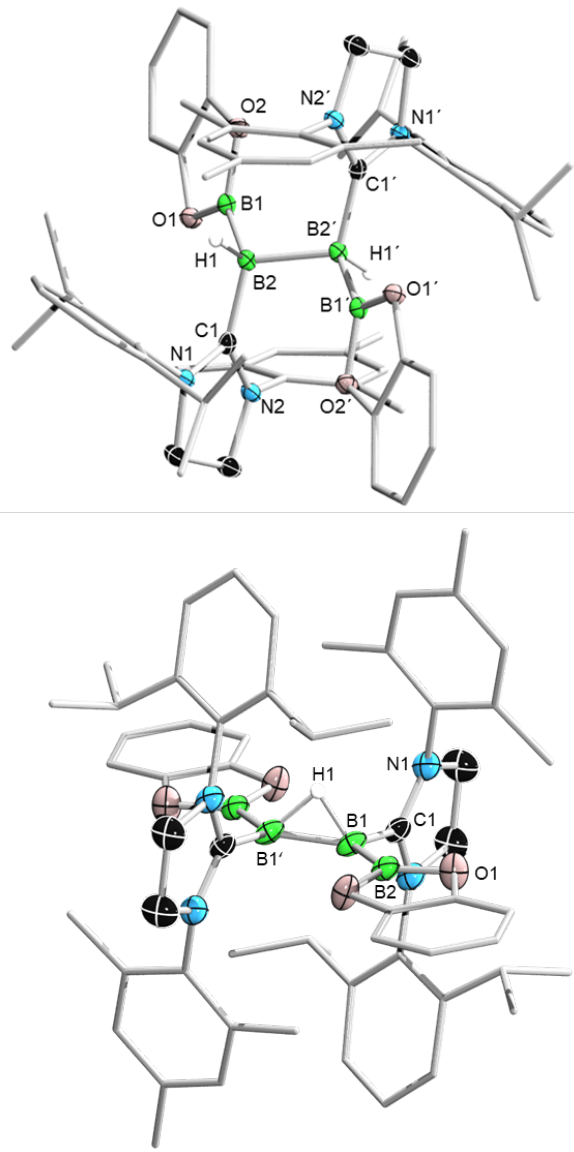

Figure 3. Molecular structures of $\mathbf{3}$ (top) and 4a (bottom) with selected atomic displacement ellipsoids at the $50 \%$ probability level. All hydrogen atoms except those bound to boron are omitted for clarity. Selected bond lengths $(\AA)$, angles and torsion angles $\left(^{\circ}\right)$ : Compound 3: B1-B2 1.679(3), B2-B2' 1.842(2), B2-C1 1.585(2), B2-H1 1.14(2), B1-B2-B2' 110.6(1), B2'-B2-C1 109.6(1), B1-B2C1 116.9(1). Compound 4: B1-B1' 1.635(5), B1-B2 1.699(5), B1C1 1.588(6), B2-B1-B1' 121.9(3), C1-B1-B2 121.7(3), B2-B1B1'-B2' 180.0(3), O1-B2-B1-B1' 170.8(4).

These observations concur with investigations by Tamao and co-workers on the compound $\operatorname{EindB}(\mu-\mathrm{H})_{2}$ BEind (Eind $=$ $1,1,3,3,5,5,7,7$-tetraethyl- $s$-hydrindacen-4-yl; $\left.\mathrm{WBI}_{\mathrm{B}-\mathrm{B}}=1.533\right)$, for which one resonance description is a doubly protonated boron-boron triple bond. ${ }^{37}$ In the case of 4 , the B1/B1' NPA values are more negative, presumably as a result of $\sigma$-donation from both the boryl substituents and the flanking carbene ligands.

Having access to diborene $\mathbf{5}$ via a previously published route, ${ }^{14}$ we attempted to synthesize 4 via protonation (Scheme 4). Treatment of 5 with one equivalent of triflic acid (HOTf) in $\mathrm{CD}_{2} \mathrm{Cl}_{2}$ resulted in clean conversion to compound $\mathbf{4}$ as judged by NMR spectroscopy. This result adds to a growing body of work ${ }^{17,38-39}$ showing that judicious choice of substituents allows electronrich boron compounds to shuttle $\mathrm{H}^{+}$rather than $\mathrm{H}^{-}$.

Scheme 4. Access to 4 via protonation of diboryldiborene 5 .

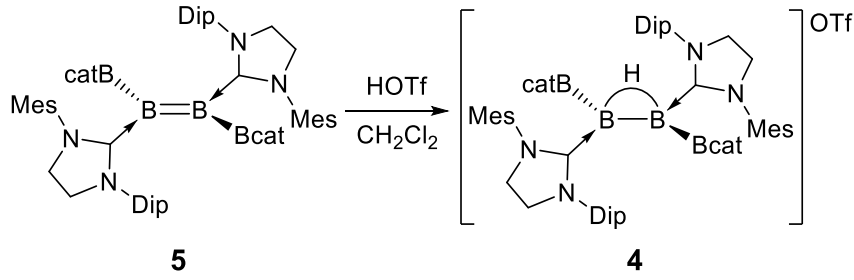

Further addition of MeOTf to 4 led to no reaction, which is consistent with the bridging hydrogen atom having a partial positive charge and thus being unwilling to be abstracted as $\mathrm{H}^{-}$. Whereas neutral, H-bridged diboranes(5) have been shown to coordinate $\mathrm{Cu}(\mathrm{I})$ fragments, ${ }^{34}$ compound $\mathbf{4}$ showed no reactivity towards $\mathrm{CuCl}$, probably as a result of significant electrostatic repulsion between the cationic species and the copper centre.

CONCLUSIONS In summary, we have described the first hydroborations of diborynes, yielding an unsymmetrical diborene and a neutral dihydrotetraborane. Abstraction of one hydride led to a cationic, hydride-bridged tetraborane that shows characteristics of a protonated diborene. Our efforts are currently focused on corroborating the last remaining links between classes of base-stabilized, classically bonded diboron hydride compounds.

\section{ASSOCIATED CONTENT}

Supporting information including details of synthesis, analytical techniques and crystal structures in .cif format. This material is available free of charge via the Internet at http://pubs.acs.org.

\section{ACKNOWLEDGEMENT}

We gratefully acknowledge the European Research Council for funding under the European Union Horizon 2020 Research and Innovation Program (grant agreement no. 669054).

\section{AUTHOR INFORMATION}

\section{Corresponding Author}

* Prof. Dr. H. Braunschweig

h.braunschweig@uni-wuerzburg.de

\section{Author Contributions}

All authors have given approval to the final version of the manuscript.

\section{REFERENCES}

1. Stock, A., Hydrides of Boron and Silicon. Cornell Univ. Press: Ithaca, NY, 1933.

2. Lipscomb, W. N., Recent Studies of the Boron Hydrides. Adv. Inorg. Chem. 1959, 1, 117-156.

3. Fox, M. A.; Wade, K., Evolving Patterns in Boron Cluster Chemistry. Pure Appl. Chem. 2003, 75 (9), 1315-1323.

4. Plešek, J., Potential Applications of the Boron Cluster Compounds. Chem. Rev. 1992, 92 (2), 269-278.

5. Arrowsmith, M.; Braunschweig, H.; Stennett, T. E., Formation and Reactivity of Electron-Precise B-B Single and Multiple Bonds. Angew. Chem. Int. Ed. 2017, 56 (1), 96-115. 
6. Braunschweig, H.; Dewhurst, R. D.; Mozo, S., Building ElectronPrecise BoronBoron Single Bonds: Imposing Monogamy on a Promiscuous Element. Chemcatchem 2015, 7 (11), 1630-1638.

7. Braunschweig, H.; Dewhurst, R. D., Single, Double, Triple Bonds and Chains: The Formation of Electron-Precise BB Bonds. Angew. Chem. Int. Ed. 2013, 52 (13), 3574-3583.

8. Lu, W.; Li, Y. X.; Kinjo, R., Crystalline Tetraatomic Boron(0) Species. J. Am. Chem. Soc. 2019, 141 (13), 5164-5168.

9. Braunschweig, H.; Dewhurst, R. D.; Hörl, C.; Phukan, A. K.; Pinzner, F.; Ullrich, S., Direct Hydroboration of $\mathrm{B}=\mathrm{B}$ Bonds: $\mathrm{A}$ Mild Strategy for the Proliferation of B-B Bonds. Angew. Chem. Int. Ed. 2014, 53 (12), 3241-3244.

10. Braunschweig, H.; Hörl, C., Unexpected Cluster Formation Upon Hydroboration of a Neutral Diborene with 9-BBN. Chem. Commun. 2014, 50 (75), 10983-10985.

11. Stennett, T. E.; Bertermann, R.; Braunschweig, H., Construction of Linear and Branched Tetraboranes by 1,1-and 1,2-Diboration of Diborenes. Angew. Chem. Int. Ed. 2018, 57 (48), 15896-15901.

12. Lu, W.; Xu, K.; Li, Y. X.; Hirao, H.; Kinjo, R., Facile Activation of Homoatomic $\sigma$-Bonds in White Phosphorus and Diborane by a Diboraallene. Angew. Chem. Int. Ed. 2018, 57 (48), 15691-15695.

13. Hermann, A.; Cid, J.; Mattock, J. D.; Dewhurst, R. D.; Krummenacher, I.; Vargas, A.; Ingleson, M. J.; Braunschweig, H., Diboryldiborenes: $\pi$-Conjugated B4 Chains Isoelectronic to the Butadiene Dication. Angew. Chem. Int. Ed. 2018, 57 (32), 10091-10095.

14. Brückner, T.; Dewhurst, R. D.; Dellermann, T.; Müller, M.; Braunschweig, H., Mild Synthesis of Diboryldiborenes by Diboration of B-B Triple Bonds. Chem. Sci. DOI: 10.1039/C9SC02544H

15. Shoji, Y.; Kaneda, S.; Fueno, H.; Tanaka, K.; Tamao, K.; Hashizume, D.; Matsuo, T., An Isolable Diborane(4) Compound with Terminal B-H Bonds: Structural Characteristics and Electronic Properties. Chem. Lett. 2014, 43 (10), 1587-1589.

16. Shoji, Y.; Matsuo, T.; Hashizume, D.; Gutmann, M. J.; Fueno, H.; Tanaka, K.; Tamao, K., Boron-Boron $\sigma$-Bond Formation by TwoElectron Reduction of a H-Bridged Dimer of Monoborane. J. Am. Chem. Soc. 2011, 133 (29), 11058-11061.

17. Kaese, T.; Budy, H.; Bolte, M.; Lerner, H. W.; Wagner, M., Deprotonation of a Seemingly Hydridic Diborane(6) To Build a B-B Bond. Angew. Chem. Int. Ed. 2017, 56 (26), 7546-7550.

18. Hübner, A.; Bolte, M.; Lerner, H.-W.; Wagner, M., Extensive Structural Rearrangements upon Reduction of 9H-9-Borafluorene. Angew. Chem. Int. Ed. 2014, 53 (39), 10408-10411.

19. Kaese, T.; Hübner, A.; Bolte, M.; Lerner, H.-W.; Wagner, M., Forming B-B Bonds by the Controlled Reduction of a Tetraaryldiborane(6). J. Am. Chem. Soc. 2016, 138 (19), 6224-6233.

20. Prokofjevs, A.; Kampf, J. W.; Solovyev, A.; Curran, D. P.; Vedejs, E., Weakly Stabilized Primary Borenium Cations and Their Dicationic Dimers. J. Am. Chem. Soc. 2013, 135 (42), 15686-15689.

21. Ghadwal, R. S.; Schurmann, C. J.; Andrada, D. M.; Frenking, G., Mono- and Di-Cationic Hydrido Boron Compounds. Dalton Trans. 2015, 44 (32), 14359-14367.

22. Wang, Y.; Quillian, B.; Wei, P.; Wannere, C. S.; Xie, Y.; King, R. B.; Schaefer, H. F., III; Schleyer, P. v. R.; Robinson, G. H., A Stable Neutral Diborene Containing a $\mathrm{B}=\mathrm{B}$ Double Bond. J. Am. Chem. Soc. 2007, 129 (41), 12412-12413.

23. Muessig, J. H.; Thaler, M.; Dewhurst, R. D.; Paprocki, V.; Seufert, J.; Mattock, J. D.; Vargas, A.; Braunschweig, H., Phosphine-Stabilized Diiododiborenes: Isolable Diborenes with Six Labile Bonds. Angew. Chem. Int. Ed. 2019, 58 (13), 4405-4409.
24. DePoy, R. E.; Kodama, G., Formation and Reaction Chemistry of Trimethylamine-Trimethylphosphine-Diborane(4). Inorg. Chem. 1988, 27 (6), 1116-1118.

25. Paine, R. T., Phosphorus-Nitrogen-Boron Heteroring Systems Preparation of Methylaminobis(Difluorophosphine)Diborane(4). J. Am. Chem. Soc. 1977, 99 (11), 3884-3885.

26. Schulenberg, N.; Wadepohl, H.; Himmel, H. J., Synthesis and Characterization of a Doubly Base-Stabilized $\mathrm{B}_{3} \mathrm{H}_{6}{ }^{+}$Analogue. Angew. Chem. Int. Ed. 2011, 50 (44), 10444-10447.

27. Dömling, M.; Arrowsmith, M.; Schmidt, U.; Werner, L.; Castro, A. C.; Jiménez-Halla, J. O. C.; Bertermann, R.; Müssig, J.; Prieschl, D.; Braunschweig, H., Spontaneous trans-Selective Transfer Hydrogenation of Apolar Boron-Boron Double Bonds. Angew. Chem. Int. Ed. 2019, 58 (29), 9782-9786.

28. Yagi, A.; Kisu, H.; Yamashita, M., Synthesis of a HydrogenBridged Tetraborane(6): a Substituent Effect of a Diaminoboryl Group Toward the B-B Multiple Bond Character. Dalton Trans. 2019, 48 (17), 5496-5499.

29. Braunschweig, H.; Dewhurst, R. D.; Hammond, K.; Mies, J.; Radacki, K.; Vargas, A., Ambient-Temperature Isolation of a Compound with a Boron-Boron Triple Bond. Science 2012, 336 (6087), 1420-1422.

30. Arrowsmith, M.; Bohnke, J.; Braunschweig, H.; Celik, M. A.; Dellermann, T.; Hammond, K., Uncatalyzed Hydrogenation of FirstRow Main Group Multiple Bonds. Chem. Eur. J. 2016, 22 (48), 17169-17172.

31. Stennett, T. E.; Mattock, J. D.; Vollert, I.; Vargas, A.; Braunschweig, H., Unsymmetrical, Cyclic Diborenes and Thermal Rearrangement to a Borylborylene. Angew. Chem. Int. Ed. 2018, 57 (15), 4098-4102.

32. Lu, W.; Li, Y. X.; Ganguly, R.; Kinjo, R., Alkene-Carbene Isomerization Induced by Borane: Access to an Asymmetrical Diborene. J. Am. Chem. Soc. 2017, 139 (14), 5047-5050.

33. Bissinger, P.; Braunschweig, H.; Damme, A.; Dewhurst, R. D.; Kupfer, T.; Radacki, K.; Wagner, K., Generation of a Carbene-Stabilized Bora-borylene and its Insertion into a C-H Bond. J. Am. Chem. Soc. 2011, 133 (47), 19044-19047.

34. Wang, S. R.; Prieschl, D.; Mattock, J. D.; Arrowsmith, M.; Pranckevicius, C.; Stennett, T. E.; Dewhurst, R. D.; Vargas, A.; Braunschweig, H., Bottleable Neutral Analogues of $\left[\mathrm{B}_{2} \mathrm{H}_{5}\right]^{-}$as Versatile and Strongly Binding $\eta^{2}$ Donor Ligands. Angew. Chem. Int. Ed. 2018, 57 (21), 6347-6351.

35. Lammertsma, K.; Ohwada, T., Three-Center, Two-Electron Systems. Origin of the Tilting of Their Substituents. J. Am. Chem. Soc. 1996, 118 (31), 7247-7254.

36. Bissinger, P.; Braunschweig, H.; Damme, A.; Kupfer, T.; Krummenacher, I.; Vargas, A., Boron Radical Cations from the Facile Oxidation of Electron-Rich Diborenes. Angew. Chem. Int. Ed. 2014, 53 (22), 5689-5693.

37. Shoji, Y.; Matsuo, T.; Hashizume, D.; Fueno, H.; Tanaka, K.; Tamao, K., A Stable Doubly Hydrogen-Bridged Butterfly-Shaped Diborane(4) Compound. J. Am. Chem. Soc. 2010, 132 (24), 82588260.

38. Ruiz, D. A.; Ung, G.; Melaimi, M.; Bertrand, G., Deprotonation of a Borohydride: Synthesis of a Carbene-Stabilized Boryl Anion. Angew. Chem. Int. Ed. 2013, 52 (29), 7590-7592.

39. Landmann, J.; Keppner, F.; Hofmann, D. B.; Sprenger, J. A. P.; Häring, M.; Zottnick, S. H.; Müller-Buschbaum, K.; Ignat'ev, N. V.; Finze, M., Deprotonation of a Hydridoborate Anion. Angew. Chem. Int. Ed. 2017, 56 (10), 2795-2799. 
Table of Contents artwork

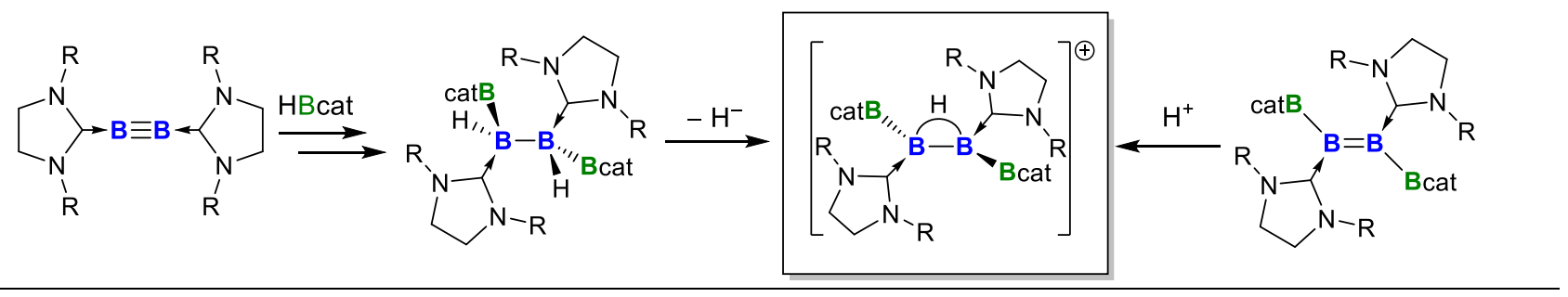

